Originalien

Chirurg 2020 $91: 337-344$

https://doi.org/10.1007/s00104-019-01054-0

Online publiziert: 25. Oktober 2019

(C) Der/die Autor(en) 2019

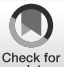

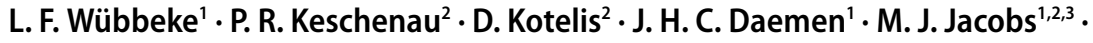
B. M. E. Mees ${ }^{1,3}$

${ }^{1}$ Klinik für Gefäßchirurgie, Universitätsklinik Maastricht, Maastricht, Niederlande

${ }^{2}$ Klinik für Gefäßchirurgie, Universitätsklinikum der RWTH Aachen, Aachen, Deutschland

${ }^{3}$ Europäisches Gefäßzentrum Aachen-Maastricht, Deutschland - Niederlande, Maastricht, Niederlande

\title{
Oberschenkelmuskellappen bei inguinalen postoperativen Komplikationen in der Gefäßchirurgie
}

\author{
Musculus sartorius vs. Musculus rectus \\ femoris
}

\section{Hintergrund}

Wundkomplikationen in der Leistengegend sind ein häufiger Grund für postoperative Morbidität und auch Letalität nach gefäßchirurgischen Eingriffen [1, 2]. Trotz aller Bemühungen, Infektionen der Operationsstelle vorzubeugen, werden in der Literatur Inzidenzen von 4-43\% berichtet [3-6]. Besonders tiefe Protheseninfektionen stellen in der Gefäßchirurgie aufgrund der hohen Mortalitäts- und Amputationsraten zwischen 6-75\% und 22-75\% eine der am meisten gefürchteten Komplikationen dar [1, 2].

Die Leistenbeuge ist aus verschiedenen Gründen ein ungünstiges Wundumfeld und die am häufigsten von postoperativen Infektionen befallene Region in der Gefäßchirurgie [2, 7, 8]. Die bekannteste Einteilung postoperativer Wundinfektionen geht auf Szilagy zurück und beschreibt drei Kategorien [9]. Grad I umfasst eine oberflächliche Infektion begrenzt auf die Haut, wohingegen sich eine Grad-II-Infektion auch auf das subkutane Gewebe erstreckt. Eine GradIII-Infektion betrifft letztendlich auch die Gefäßprothese und führt zu einem erhöhten Risiko einer Bakteriämie [9]. Der traditionelle chirurgische Therapieansatz umfasst die Verabreichung systemischer Antibiotika und die Entfernung der infizierten Gefäßprothese, gefolgt von einer erneuten Rekonstruktion falls erforderlich [9-11]. Seit 1976 wird die Bedeckung der Leistengegend mit einem Oberschenkelmuskellappen als zusätzliches Therapieverfahren beschrieben, da auch beim traditionellen Therapieansatz hohe Morbiditäts- und Mortalitätszahlen berichtet werden [8, 12-14]. Diese Muskellappen decken die großen Gewebedefekte $a b$, die durch die Infektion und chirurgisches Débridement entstehen. Außerdem hat die Muskellappenplastik aufgrund eines erhöhten Blutflusses mit erhöhter Zufuhr von Sauerstoff, Nährstoffen und Antibiotika in den infizierten Bereich positive Auswirkungen auf den Heilungsprozess [7, 14]. In der Literatur werden verschiedene Muskellappen zur Wundrekonstruktion in der Leistengegend beschrieben. Weltweit ist die Sartorius-Muskellappen-Schwenkplastik das am häufigsten verwendete und beschriebene Verfahren. Die Nutzung des Rectus-femoris-Muskellappens ist jedoch ein vielversprechendes Alternativverfahren mit mehreren Vorteilen. Trotz des zweiten Weichteildefekts, der für die Entnahme des Rectus-femorisMuskellappens erforderlich ist, sind das größere Volumen des Muskels und der größere Abstand zum betroffenen infizierten Bereich in der Leiste in erster Linie günstig für die Behandlung komplexer Leistenwundinfektionen. Darüber hinaus ist die Mobilisierung des Sartorius-Lappens aufgrund der segmentalen Blutversorgung durch die oberflächliche Femoralarterie, welche bei Patienten mit Atherosklerose außerdem häufig selbst geschädigt ist, nur von lateral möglich. Der Rectus-femoris-Muskellappen verfügt über eine zuverlässigere Blutversorgung durch die Arteria profunda femoris [7, 8, 14, 15]. Die Entscheidung, welcher Muskellappen zur Bedeckung der Leiste entnommen wird, hängt hauptsächlich von der Präferenz und Erfahrung des Krankenhauses und des operierenden Chirurgen ab. Obwohl tiefe Wundinfektionen in der Leiste seit Jahrzehnten eine schwierige und häufige Komplikation sind, ist die optimale therapeutische Strategie immer noch sehr unklar. Ein randomisierter Vergleich zwischen dem Sartorius-Muskellappen und dem Rectus-femoris-Muskellappen wurde nie durchgeführt [7].

Ziel dieser Studie war es daher, von unseren Erfahrungen mit der Anwendung des Rectus-femoris-Muskellappens und des Sartorius-Muskellappens bei Komplikationen der tiefen Leistengegend in der Gefäßchirurgie zu berichten.

\section{Material und Methoden}

Dies ist eine retrospektive monozentrische Studie. Wir analysierten alle Pati- 

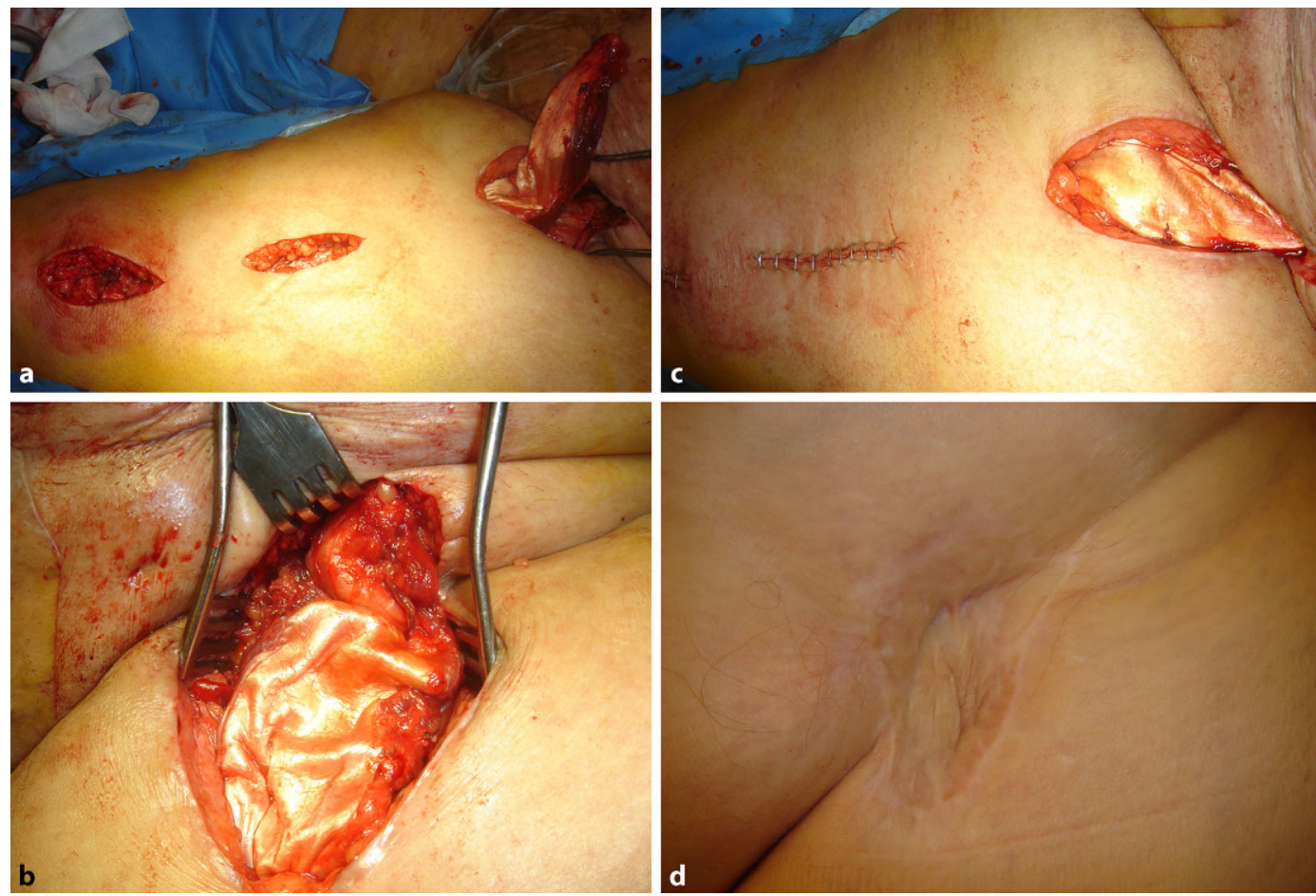

Abb. $1 \triangleleft$ Darstellung einer Muskellappenplastik mit dem Musculus rectus femoris. a Zusätzliche Längsschnitte in der Mitte des Oberschenkels. b Der Muskellappen wird über einen subkutanen Tunnel zur Leistenwunde geführt, um die Gefäßprothese abzudecken. c Der Muskellappen füllt den entstandenen Weichteildefekt. Die Entnahmestellen werden mit einem geschichteten Wundverschluss versorgt. d Eine vollständige Wundheilung wurde erreicht

enten, die im Zeitraum zwischen 2006 und 2017 an den zwei Standorten eines grenzüberschreitenden Gefäßzentrums mit einer der beiden Muskellappenplastiken behandelt wurden. Vor Beginn dieser Studie wurde die Zustimmung der örtlichen Ethikkommission eingeholt. Patienten, die aus anderen Gründen als Gefäßoperationen eine Muskellappenplastik in der Leiste bekamen oder mit anderen Muskellappen behandelt wurden, wurden ausgeschlossen.

\section{Datenerhebung}

Wie bereits erwähnt, wurden alle Daten rückwirkend erhoben. Die elektronischen Patientenakten der Krankenhäuser wurden verwendet, um demographische Daten sowie Begleiterkrankungen zu identifizieren. Darüber hinaus wurden verschiedene operative Merkmale und klinische Ergebnisse erhoben. Alle Amputationen über dem Sprunggelenk wurden als Majoramputation und daher als Gliedmaßenverlust definiert. Es wurde zwischen primärem und sekundärem Verlust der Gefäßprothese unterschieden. Sekundärer Verlust der Gefäßprothese trat auf, wenn eine Reintervention zur sekundären Gefäßprothesenentnahme erforderlich war.

\section{Operationstechniken}

Alle analysierten Muskellappenoperationen wurden durch Gefäßchirurgen ausgeführt. Die Leistenwunden wurden in der Regel mikrobiologisch untersucht und eine Antibiotikatherapie gestartet. Die Wunden wurden ausgiebig chirurgisch gesäubert und gespült. Die Auswahl des Muskellappens wurde vom Operateur getroffen und basierte immer auf einer Beurteilung der Wundgröße und des entstandenen Weichteildefekts sowie der Beurteilung der Eignung der lokalen Muskellappen. Die Wunden wurden subjektiv beurteilt und es wurden keine Volumenmessungen oder spezifische Untersuchungen der Blutversorgung durchgeführt.

Um den Rectus-femoris-Muskel zu entnehmen, wird ein zusätzlicher Längsschnitt in der Mitte des Oberschenkels vorgenommen, der sich über die distalen zwei Drittel des Oberschenkels erstreckt (•Abb. 1). Der Musculus rectus femoris wird identifiziert und von seiner distalen Anheftung an der Patella sowie von seinen Faszienverbindungen gelöst. Die anführende laterale Femoralarterie wird identifiziert und erhalten. Der Muskellappen wird in einer distalproximalen Vorgehensweise angehoben und durch einen subkutanen Tunnel, der die Entnahmestelle und die Leistenwunde verbindet, in die Leistenwunde eingeführt. Abhängig vom Gewebedefekt wird die Wunde schichtweise über eine Saugdrainage geschlossen oder eine Vacuum-assisted closure(VAC)Therapie eingeleitet. Die Entnahmestelle wird ebenfalls mit einem geschichteten Wundverschluss versorgt. Einschließlich ausgiebigem Säubern und Spülen der Wunde dauert eine Rectus-femorisMuskellappenplastik im Allgemeinen 30-60 min.

Für die Sartorius-Muskellappenplastik wird die Inzision der Leistenwunde zunächst nach lateral verlängert und die Faszie eingeschnitten, wodurch der Sartorius-Muskel freigelegt werden kann. Der proximale Rand des Muskels wird abgelöst und die segmentalen Äste der A. femoralis superficialis an der Unterseite des Muskels werden identifiziert. Der Muskel wird umgedreht und in die Leistenwunde gelegt. Wieder wird entweder eine VAC-Therapie eingeleitet oder die Wunde schichtweise über eine Saugdrainage geschlossen. Einschließlich Säubern und Spülen wird auch die Sartorius-Muskellappenplastik im Allgemeinen innerhalb von 30-60 min durchgeführt. 
Chirurg 2020 -91:337-344 https://doi.org/10.1007/s00104-019-01054-0

(c) Der/die Autor(en) 2019

L. F. Wübbeke · P. R. Keschenau · D. Kotelis · J. H. C. Daemen · M. J. Jacobs · B. M. E. Mees

\section{Oberschenkelmuskellappen bei inguinalen postoperativen Komplikationen in der Gefäßchirurgie. Musculus sartorius vs. Musculus rectus femoris}

\section{Zusammenfassung}

Hintergrund. Wundkomplikationen in der Leiste verursachen häufig postoperative Morbidität und auch Letalität nach gefäßchirurgischen Eingriffen. Ziel dieser Studie war es, von unseren Erfahrungen mit einer Rectus-femoris-Muskellappenplastik (RFF) im Vergleich zur Sartorius-Muskellappenplastik (SMF) zu berichten.

Material und Methoden. Eine retrospektive Studie wurde an zwei Standorten eines grenzüberschreitenden Gefäßzentrums durchgeführt und alle in der Gefäßchirurgie durchgeführten Muskellappenplastiken wurden untersucht. Primäre Endpunkte waren das Überleben der Muskellappen, Erhaltung der Gefäßprothese und Majoramputationen.
Ergebnisse. Insgesamt wurden 44 Rectusfemoris-Plastiken bei 39 Patienten (mittleres Alter 67 Jahre, $73 \%$ Männer) und 25 SartoriusPlastiken bei 24 Patienten (mittleres Alter 64 Jahre, $76 \%$ Männer) durchgeführt. Wundinfektionen waren die häufigste Indikation für die Muskellappenplastik. Während eines durchschnittlichen Follow-up-Zeitraums von 24 ( \pm 24$)$ bzw. 17 Monaten $( \pm 20)$ wurden vergleichbare Überlebensraten der Muskellappen (91\% vs. $84 \%$ ), Wundheilungsraten ( $72 \%$ vs. $83 \%)$, Prothesenerhalt ( $65 \%$ vs. $73 \%$ ) und Amputationsraten (9\% vs. $8 \%$ ) gefunden. Schlussfolgerung. Die Muskellappenplastik ist ein effektives Verfahren, um Leistendefekte durch tiefe Infektionen nach gefäßchirurgischen Eingriffen zu bedecken, mit guten
Ergebnissen in einer "Hochrisiko"-Patientengruppe. Es wurden keine Unterschiede zwischen beiden Muskellappen in Bezug auf Amputationen und Verlust der Gefäßprothese gefunden. Beide Techniken können sicher durchgeführt werden, je nach Präferenz und Erfahrung des Operationsteams, wobei die Rectus-femoris-Muskellappenplastikvor allem bei großen Gewebedefekten zu empfehlen ist und eine Sartorius-Muskellappenplastik für kleinere Defekte.

Schlüsselwörter

Wundinfektionen - Protheseninfektionen . Muskellappenplastik · M. sartorius · M. rectus femoris

\section{Thigh muscle flaps for postoperative inguinal wound complications in vascular surgery. Sartorius muscle versus rectus femoris muscle}

\section{Abstract}

Background. Inguinal wound complications often cause postoperative morbidity and also mortality following vascular surgical interventions. The aim of this study was to report experiences and a comparison of the outcomes using rectus femoris muscle flaps (RFF) and sartorius muscle flaps (SMF).

Material and methods. A retrospective study was performed at two locations of a crossborder vascular center and all muscle flap interventions performed at the two centers within the vascular surgery department were reviewed. Primary outcomes were muscle flap survival, graft salvage and major amputations.
Results. A total of 44 RFFs were performed in 39 patients (mean age 67 years, $73 \%$ males) and 25 SMFs in 24 patients (mean age 64 years, $76 \%$ males). Wound infections were the most common indications for muscle flap reconstruction. At a mean follow-up of 24 months $( \pm 24)$ and 17 months $( \pm 20)$, respectively, comparable flap survival rates $(91 \%$ vs. $84 \%)$, wound healing rates $(72 \%$ vs. $83 \%)$, graft salvage ( $65 \%$ vs. $73 \%)$ and amputation rates ( $9 \%$ vs. $8 \%$ ) were found. Conclusion. Muscle flap reconstruction is an effective way to cover groin defects resulting from deep wound infections after vascular surgery, achieving good results in a high-risk group of patients. No differences were found between SMF and RFF regarding amputation and graft loss. Both techniques can be safely performed, depending on the preference and experience of the surgical team. The RFF technique should be preferentially used to cover large tissue defects, whereas the SMF procedure can be preferred to cover smaller defects in the groin.

Keywords

Surgical wound infection - Vascular prosthesis infection - Groin wound reconstruction . Rectus femoris muscle · Sartorius muscle
Postoperativ wurde die initiierte Antibiotikatherapie basierend auf den mikrobiologischen Ergebnissen angepasst und in der Regel über mehrere Wochen, zunächst intravenös und dann in oraler Form, fortgesetzt. Diese Entscheidungen wurden immer in enger Zusammenarbeit mit Infektiologen getroffen. Die Patienten konnten in der Regel nach wenigen Tagen wieder mobilisiert werden und das Krankenhaus nach ausreichender weiterer Wundheilung, sodass eine ambulante Wundversorgung möglich ist, verlassen. Regelmäßige Wundkontrollen fanden zunächst im stationären und später im ambulanten Umfeld statt bis die Leistenwunden verheilt waren.

\section{Endpunkte der Studie}

Die primären Endpunkte waren das Überleben der Muskellappen, Erhaltung der Gefäßprothese und Majoramputationen. Sekundäre Endpunkte waren Wundheilung, lokale Wundkomplikationen innerhalb von 30 Tagen sowie
Komplikationen der Rectus-femorisEntnahmestelle, Gefäßkomplikationen und das Überleben des Patienten. Darüber hinaus wurden die Morbidität aufgrund einer gestörten Muskelkraft des Knieextensors nach Rectus-femorisEntnahme und die Dauer des Krankenhausaufenthalts als sekundäre Endpunkte analysiert. Das Datum des letzten klinischen Kontakts wurde zur Berechnung der Follow-up-Periode pro Patient verwendet. 
Tab. 1 Demographische Daten und Begleiterkrankungen ${ }^{a}$

\begin{tabular}{|c|c|c|c|}
\hline & $\begin{array}{l}\text { M. rectus femoris } \\
(n=39)\end{array}$ & $\begin{array}{l}\text { M. sartorius } \\
(n=24)\end{array}$ & $p$-Wert \\
\hline & 44 Plastiken & 25 Plastiken & \\
\hline Durchschnittsalter (Spanne) & $67 \pm 9,6(36-86)$ & $\begin{array}{l}65 \pm 16,0 \\
(26-85)\end{array}$ & 0,34 \\
\hline Alter $>80$ Jahre & $7 \%$ & $20 \%$ & 0,13 \\
\hline Geschlecht & & & 0,77 \\
\hline Weiblich & $27 \%$ & $24 \%$ & \\
\hline Männlich & $73 \%$ & $76 \%$ & \\
\hline \multicolumn{4}{|c|}{ Risikofaktoren und Begleiterkrankungen } \\
\hline Diabetes mellitus & $36 \%$ & $36 \%$ & 0,98 \\
\hline Nikotinabusus & $43 \%$ & $60 \%$ & 0,18 \\
\hline Body-Mass-Index $\left(\mathrm{kg} / \mathrm{m}^{2}\right)$ & $25,8 \pm 5,4$ & $25,1 \pm 3,9$ & 0,58 \\
\hline Body-Mass-Index $>25 \mathrm{~kg} / \mathrm{m}^{2}$ & $58 \%$ & $48 \%$ & 0,42 \\
\hline Hypertonie & $77 \%$ & $76 \%$ & 0,94 \\
\hline Hypercholesterinämie & $86 \%$ & $44 \%$ & $<0,001$ \\
\hline Herzerkrankungen & $39 \%$ & $36 \%$ & 0,83 \\
\hline COPD & $21 \%$ & $32 \%$ & 0,29 \\
\hline Chronische Niereninsuffizienz & $5 \%$ & $44 \%$ & $<0,001$ \\
\hline \multicolumn{4}{|c|}{$\begin{array}{l}\text { 'Daten als Mittelwert } \pm \text { Standardabweichung oder in Prozent angegeben. Unterschiede zwischen } \\
\text { den beiden Behandlungsgruppen wurden mit } x^{2} \text {-Tests (bei kategorialen Daten) oder t-Tests für } \\
\text { unabhängige Stichproben (bei kontinuierlichen Daten) untersucht } \\
\text { COPD chronisch-obstruktive Lungenerkrankungen }\end{array}$} \\
\hline
\end{tabular}

Tab. 2 Operative Merkmale ${ }^{\mathrm{a}}$

\begin{tabular}{|c|c|c|c|}
\hline & $\begin{array}{l}\text { M. rectus femoris } \\
(n=44)\end{array}$ & $\begin{array}{l}\text { M. sartorius } \\
(n=25)\end{array}$ & $p$-Wert \\
\hline Frühere Leistenoperation & $98 \%$ & $88 \%$ & 0,13 \\
\hline Anzahl Voroperationen (Spanne) & $2,6(0-6)$ & $2,3(0-6)$ & 0,50 \\
\hline $\begin{array}{l}\text { Durchschnittlicher Zeitraum zwischen der } \\
\text { letzten Leistenoperation und der Muskellap- } \\
\text { penplastik (Tage) }\end{array}$ & 322 & 96 & 0,10 \\
\hline Kunststoffmaterial vorhanden & $98 \%$ & $84 \%$ & 0,05 \\
\hline Indikation für Muskellappenplastik & & & 0,27 \\
\hline Wundinfektionen & $48 \%$ & $56 \%$ & \\
\hline Blow-out ${ }^{\text {b }}$ & $21 \%$ & $4 \%$ & \\
\hline Blutung & $2 \%$ & $12 \%$ & \\
\hline Hämatom & $5 \%$ & $8 \%$ & \\
\hline Falsches Aneurysma & $18 \%$ & $12 \%$ & \\
\hline Prophylaktisch & $7 \%$ & $8 \%$ & \\
\hline Notfalleingriff & $57 \%$ & $56 \%$ & 0,95 \\
\hline Primäre Prothesenexzision & $40 \%(n=17)$ & $48 \%(n=10)$ & 0,79 \\
\hline Gefäßabnaht ohne Rekonstruktion & 6 & 3 & \\
\hline Autologe Rekonstruktion & 5 & 3 & \\
\hline Nichtautologe Rekonstruktion & 6 & 4 & \\
\hline Vacuum-assisted closure therapy & $56 \%$ & $81 \%$ & 0,05 \\
\hline Positive Wundkulturen & $64 \%$ & $70 \%$ & 0,48 \\
\hline \multicolumn{4}{|c|}{$\begin{array}{l}{ }^{a} \text { Daten als Mittelwert oder als Prozentsatz dargestellt. Unterschiede zwischen den beiden Be- } \\
\text { handlungsgruppen wurden mit } x^{2} \text {-Tests (bei kategorialen Daten) oder t-Tests für unabhängige } \\
\text { Stichproben (bei kontinuierlichen Daten) untersucht } \\
{ }^{b} \text { Anastomosenblutung durch Blow-out der Anastomose }\end{array}$} \\
\hline
\end{tabular}

\section{Statistische Analyse}

Alle statistischen Analysen wurden mit SPSS Statistics 24 durchgeführt und die beiden Behandlungsgruppen (M. rectus femoris vs. M. sartorius) wurden miteinander verglichen. Für die demographischen Daten und Begleiterkrankungen wurden Standardverfahren der deskriptiven Statistik verwendet. Kategoriale Daten wurden als Prozentsätze dargestellt, kontinuierliche Daten als Mittelwert in Kombination mit der Standardabweichung (SD). Die Unterschiede zwischen beiden Gruppen wurden mit $\chi^{2}$-Tests bewertet. Um die Mittelwerte der kontinuierlichen Variablen zu vergleichen, wurden $t$-Tests für unabhängige Stichproben verwendet. Als statistisch signifikant wurden $p$-Werte von $<0,05$ angesehen.

\section{Ergebnisse}

Während des Untersuchungszeitraums wurden bei 39 Patienten insgesamt 44 Rectus-femoris-Muskellappenplastiken und bei 24 Patienten insgesamt 25 Sartorius-Muskellappenplastiken durchgeführt. Der durchschnittliche Follow-up-Zeitraum betrug in der Rectusfemoris-Gruppe 24 Monate $( \pm 24)$ und in der Sartorius-Gruppe 17 Monate $( \pm 20)$. Die demographischen Daten aller Patienten sind in $\bullet$ Tab. 1 und die operativen Merkmale in - Tab. 2 dargestellt.

In beiden Gruppen waren mehr als zwei Drittel der Patienten männlich (73\% bzw. 76\%). Das Durchschnittsalter betrug in der Rectus-femoris-Gruppe 67 Jahre $( \pm 9,6)$ und in der SartoriusGruppe 65 Jahre $( \pm 16,0)$. Die untersuchten Begleiterkrankungen waren häufig in dieser Studienpopulation vorhanden und vereinzelt wurden signifikante Unterschiede zwischen beiden Gruppen gefunden (•Tab. 1). In der Rectus-femoris-Gruppe wurden mehr Patienten wegen Hypercholesterinämie behandelt ( $86 \%$ vs. $44 \%, p<0,001)$, während in der Sartorius-Gruppe mehr Patienten an einer chronischen Niereninsuffizienz litten ( $5 \%$ vs. $44 \%, p<0,001)$. 
Tab. 3 Klinische Ergebnisse ${ }^{a}$

\begin{tabular}{|c|c|c|c|}
\hline & $\begin{array}{l}\text { M. rectus femoris } \\
(n=44)\end{array}$ & $\begin{array}{l}\text { M. sartorius } \\
(n=25)\end{array}$ & $p$-Wert \\
\hline Überleben des Muskellappens & $91 \%$ & $84 \%$ & 0,45 \\
\hline Erhalt der Gefäßprothese & $65 \%$ & $73 \%$ & 0,52 \\
\hline Majoramputationen & $9 \%$ & $8 \%$ & 1,00 \\
\hline \multicolumn{4}{|l|}{ Mortalität } \\
\hline 30 Tage & $9 \%$ & $12 \%$ & 0,70 \\
\hline $1 \mathrm{Jahr}$ & $27 \%$ & $26 \%$ & 0,94 \\
\hline \multicolumn{4}{|l|}{ Lokale Wundkomplikationen (30 Tage) } \\
\hline Rezidivierende Wundinfektion & $34 \%$ & $12 \%$ & 0,05 \\
\hline Blutung & $12 \%$ & $8 \%$ & 1,00 \\
\hline Serom & $7 \%$ & $0 \%$ & 0,55 \\
\hline Lappennekrose & $9 \%$ & $4 \%$ & 0,65 \\
\hline Komplette Wundheilung & $72 \%$ & $83 \%$ & 0,30 \\
\hline Vaskuläre Komplikationen (30 Tage) ${ }^{b}$ & $16 \%$ & $20 \%$ & 0,75 \\
\hline Komplikationen der Entnahmestelle (30 Tage) & $2 \%$ & N.u. & \\
\hline Wundinfektion & 1 & & \\
\hline Verringerte Muskelkraft & $4 \%$ & N.u. & \\
\hline Krankenhausaufenthalt (Tage) & $27 \pm 20$ & $41 \pm 26$ & 0,02 \\
\hline $\begin{array}{l}\text { Durchschnittlicher Follow-up-Zeitraum [Mo- } \\
\text { nate (Spanne)] }\end{array}$ & $24 \pm 24(0-83)$ & $17 \pm 20(0-73)$ & 0,27 \\
\hline \multicolumn{4}{|c|}{$\begin{array}{l}\text { ¿Daten sind als Mittelwert oder als Prozentsatz dargestellt. Unterschiede zwischen den beiden } \\
\text { Behandlungsgruppen wurden mit X²-Tests (bei kategorialen Daten) oder t-Tests bei unabhängigen } \\
\text { Stichproben (bei kontinuierlichen Daten) untersucht } \\
\text { bevaskularisation jeder Art aufgrund (erneuter) kritischer Extremitätenischämie } \\
\text { N.u. Nicht untersucht }\end{array}$} \\
\hline
\end{tabular}

\section{Operative Merkmale}

In Bezug auf die operativen Merkmale hatten fast alle Patienten in dieser Studienpopulation eine Vorgeschichte mit früheren Leistenoperationen (98 und $88 \%$ ). In den meisten Leistenwunden befand sich Kunststoffmaterial ( $98 \%$ vs. $84 \%$ ). Tiefe Wundinfektionen in der Leiste waren in beiden Gruppen die häufigste Indikation für die Muskellappenplastik (48 und 56\%). Mehr als die Hälfte aller Patienten benötigte einen Notfalleingriff ( 57 und $56 \%$ ). In beiden Gruppen war mehr als die Hälfte aller Wundkulturen positiv für Mikroorganismen (64 und $72 \%$ ). Während der Rectus-femoris-Operationen wurde die Gefäßprothese aus $17 \mathrm{der}$ 43 Leisten mit Kunststoffmaterial in situ (40\%) entfernt. In 6 dieser Fälle wurde die Prothese ohne Rekonstruktion entfernt, in 5 Fällen wurde das nichtautologe Prothesenmaterial durch venöses Material ersetzt, und in den verbleibenden 6 Fällen wurde erneut nichtautologes Material verwendet. Während der Sartori-
us-Operationen wurde die ursprüngliche Gefäßprothese aus 10 der 21 Leisten mit Kunststoffmaterial in situ (48\%) entfernt, in 3 Fällen durch venöses Material ersetzt und in 4 Fällen durch Kunststoffmaterial. Mehr als die Hälfte aller Patienten benötigte eine VAC-Therapie (56 und $81 \%$ ).

\section{Endpunkte der Studie}

Die klinischen Ergebnisse nach den Muskellappenplastiken sind in - Tab. 3 dargestellt. Während des durchschnittlichen Follow-up-Zeitraums von 24 bzw. 17 Monaten ( \pm 24 bzw. 20) blieben $91 \%$ bzw. $84 \%$ aller Muskellappen vital. Wundheilung, Verlust der Gefäßprothese, Amputationen und Mortalität waren ebenfalls zwischen beiden Behandlungsgruppen vergleichbar. Insgesamt konnten $65 \%$ bzw. $73 \%$ der verbleibenden Gefäßprothesen sekundär erhalten werden. In der Rectus-femoris-Gruppe war nur eine Amputation innerhalb der ersten 30 Tage erforderlich und 4 Patienten verstarben aufgrund rezidivierender Infektion und
Sepsis. Auch in der Sartorius-Gruppe war eine Amputation innerhalb der ersten 30 Tage erforderlich. Ein Patient verstarb aufgrund von Blutungen und ein anderer Patient aufgrund einer unabhängigen Todesursache. In beiden Gruppen wurden zurückkehrende Wundinfektionen am häufigsten als lokale Komplikation an der Leistenwunde beschrieben (34\% vs. $12 \%)$. Interessanterweise war die Komplikationsrate der Entnahmestelle in der Rectus-femoris-Gruppe sehr niedrig. Nur ein Patient entwickelte eine Komplikation an der Entnahmestelle (2\%) und in nur einem Fall wurde von einer Beeinträchtigung der Muskelkraft berichtet. Die durchschnittliche Dauer des Krankenhausaufenthalts war in der Rectus-femoris-Gruppe deutlich kürzer als in der Sartorius-Gruppe $(27 \pm 20$ Tage vs. $41 \pm 26$ Tage, $p=0,02$ ).

\section{Diskussion}

Die Behandlung von Komplikationen in der Leistenwunde nach gefäßchirurgischen Eingriffen stellt weltweit immer noch eine Herausforderung für Gefäßchirurgen dar [16]. Diese retrospektive Studie an zwei Standorten eines grenzüberschreitenden Gefäßzentrums vergleicht die Ergebnisse der Rectusfemoris-Muskellappenplastik mit den Ergebnissen der Sartorius-Muskellappenplastik. Wir konnten zeigen, dass eine Muskellappenplastik ein effektives Verfahren ist, um tiefe Defekte nach Infektionen der Leistenwunde zu bedecken, und haben mit beiden Techniken gute Ergebnisse hinsichtlich Amputationen und Gefäßprothesenerhalt nachweisen können.

In dieser Studie mit einem durchschnittlichen Follow-up-Zeitraum von 2 Jahren waren $91 \%$ aller Rectus-femoris-Muskellappen und $84 \%$ aller Sartorius-Muskellappen beim letzten Patientenkontakt vital. In beiden Behandlungsgruppen traten schwerwiegenden Komplikationen wie Verlust der Gefäßprothese, Amputationen und Mortalität selten auf. Darüber hinaus benötigten mehr als die Hälfte aller Patienten einen Notfalleingriff, was den Schweregrad der Infektionen und der klinischen Darstellungen deutlich macht. Patienten mit 
tiefen Infektionen in der Leiste stellen für den Gefäßchirurgen eine schwierige Gruppe von Patienten mit schlechter Prognose dar [1, 2]. Unsere Studie zeigt, dass die Muskellappenplastik mit beiden Muskeln zu akzeptablen Mortalitätsund Amputationsraten führte, die im Vergleich mit den Mortalitäts- und Morbiditätsraten nach traditionellem Wundmanagement niedrig waren [11]. Der einzige signifikante Unterschied zwischen beiden Gruppen war eine kürzere Dauer des Krankenhausaufenthalts in der Rectus-femoris-Gruppe. Dieser Unterschied ist am wahrscheinlichsten auf allgemeine Unterschiede im Gesundheitswesen zwischen Deutschland und den Niederlanden zurückzuführen. Die Verwendung des Rectus-femoris-Muskellappens wird im niederländischen Teil des Gefäßzentrums präferiert, während die Sartorius-Muskellappenplastiken in Aachen durchgeführt wurden. Statistiken zeigen, dass die durchschnittliche Verweildauer im Jahre 2016 in deutschen Krankenhäusern 7 Tage betrug und in den Niederlanden 5 Tage $[17,18]$. Daher ist es nicht verwunderlich, dass dieser Trend auch in unserer Studie zu sehen ist.

\section{Infektionen der Leistenwunde}

Es ist nicht überraschend, dass die Leiste die Region ist, die am häufigsten von postoperativen Infektionen betroffen ist [8]. Die Mehrheit der peripheren Gefäßinterventionen wird über einen inguinalen Zugang durchgeführt. Es ist jedoch bekannt, dass die Leistenbeuge aufgrund der lymphatischen Anatomie, des Potenzials für einen großen Gewebedefekt und postoperativer Fettgewebs- und Hautnekrosen ein eher ungünstiges Wundumfeld ist $[2,7]$. Außerdem trägt auch die direkte Nähe dieses Gebietes zur stark kontaminierten Anogenitalregion zu einem erhöhten Risiko für Wundinfektionen bei [7, 8]. Darüber hinaus sind bei gefäßchirurgischen Patienten häufig mehrere Begleiterkrankungen vorhanden, wie z. B. Diabetes mellitus, die zusätzliche Risikofaktoren für Wundinfektionen darstellen [2, 7]. Diabetes, Nikotinabusus und ein hoher Body-Mass-Index (BMI) waren auch in unsere Studienpopulation häufig vorhanden, was das Risiko von Wundinfektionen erhöhte. Dies könnte mit zu der relativ hohen Rate lokaler Komplikationen nach der Muskellappenplastik beigetragen haben. Am häufigsten wurden zurückkehrende Infektionen und Blutungen berichtet, die jedoch bei der Mehrzahl der Patienten nicht zu einem Muskellappenverlust führten. Darüber hinaus werden in der Literatur vergleichbare Komplikationsraten berichtet. Mirzabeigi et al. führten eine relativ große Muskellappenstudie in den USA durch und die Autoren berichteten von einer Komplikationsrate von 34\% [7]. Obwohl in unserer Studie in der Rectus-femorisGruppe eine Tendenz zu einer höheren Komplikationsrate gefunden wurde, war der Unterschied nicht statistisch signifikant und die langfristigen klinischen Erfolge in beiden Behandlungsgruppen vergleichbar.

\section{Ergebnisse der Muskellappen- plastiken}

In der Literatur konzentrierten sich bisher wenige Studien auf die Ergebnisse der Rectus-femoris-Muskellappenplastik bei tiefen Leistenwunden. Mirzabeigi et al. berichteten von Amputationen in $5,8 \%$ der Fälle und 21,6\% Gefäßprothesenverlust [7]. Fischer et al. berichteten von Amputations- und Prothesenverlustraten von 1,4\% bzw. 2,9\% [13]. Andere Amputationsraten reichen bis zu 25\% und Prothesenverlustraten bis zu 35\% $[19,20]$. Mirzabeigi et al. berichteten außerdem von vergleichbaren Komplikationsraten, auch nachdem der Sartorius-Muskel verwendet wurde, während Fischer et al. über eine signifikant niedrigere Gesamtrate von Komplikationen von 2,9\% (Prothesenverlust, Amputation oder Reintervention) berichteten, wenn der Rectus-femoris-Muskel verwendet wurde, verglichen mit 13,2\% nach Verwendung des Sartorius-Muskels [7, 13]. In unserer Studie waren keine Unterschiede zwischen beiden Behandlungsgruppen bezüglich der primären Endpunkte zu verzeichnen. Interessanterweise war die in unserer Studie festgestellte Komplikationsrate der Rectusfemoris-Entnahmestelle hingegen sehr niedrig. Im Vergleich dazu haben Fischer et al. in ihrer Studie von einer Komplikationsrate von $7,5 \%$ berichtet [13]. Das mögliche Risiko von zusätzlicher Morbidität durch den zweiten Weichteildefekt sowie von Funktionsdefiziten der Knieextension nach der Rectus-femorisMuskellappenplastik ist einer der größten potenziellen Nachteile. Unsere Studie zeigt, dass eine zusätzliche Morbidität aufgrund der Entnahme des Muskels eine Seltenheit war. Darüber hinaus haben auch Sbitany et al. gezeigt, dass die Muskelstärke in ihrer Studie nach vollzogener Rectus-femoris-Muskellappenplastik nicht merklich abgenommen hat [21]. Weitere Studien fanden ebenfalls keine signifikante funktionelle Einschränkung [22, 23]. Ein Nachteil der Sartorius-Muskellappenplastik ist die komplexere Blutversorgung. Der M. sartorius wird segmental von der A. femoralis superficialis (SFA) durchblutet und im proximalen Teil des Muskels auch von anderen Gefäßen in diesem Bereich [24, 25]. Bei Patienten mit peripherer arterieller Verschlusskrankheit ist die SFA häufig beeinträchtigt $[7,8,14,15]$. Töpel et al. haben jedoch den Einfluss einer SFA-Okklusion auf das Ergebnis der Sartorius-Muskellappenplastik beurteilt und kein erhöhtes Risiko auf einen Verlust des Muskellappens festgestellt [26]. Viel wichtiger ist, dass das Risiko einer Muskellappennekrose erhöht ist, wenn die posteromedial eintretenden Gefäßstiele während der Mobilisierung des Sartorius-Muskellappens nicht erhalten bleiben. Um eine Lappennekrose zu vermeiden, sollte nur ein kleines Segment des M. sartorius lateral mobilisiert und dann in die Leistenwunde gedreht werden [25].

Alternative Verfahren zur plastischchirurgischen Defektdeckung in der Leiste, wie der Gracilis-Muskellappen, der Tensor-fasciae-latae(TFL)-Lappen oder der kontralaterale Rectus-abdominisLappen, können auch nach gefäßchirurgischen Eingriffen angewandt werden [27-30]. Validierte klinische Daten zu jeglichen Formen der Defektdeckung in der Leiste sind jedoch sehr begrenzt, meist basierend auf Fallberichten und Studien mit kleinen Patientengruppen. Durch Dua et al. wurde vor kurzem eine Gracilis-Muskellappen-Technik evalu- 
iert mit $100 \%$ Wundheilung als Ergebnis, sodass geschlussfolgert wurde, dass dieses Verfahren als primäres Verfahren der Defektdeckung in der Leistengegend in Erwägung gezogen werden sollte [27]. Die Rectus-femoris- und SartoriusMuskellappenplastik bieten außerdem vor allem im Vergleich zum TFL- und kontralateralen Rectus-abdominis-Lappen den Vorteil einer geringeren Invasivität, dass sie üblicherweise durch die Gefäßchirurgen selbst angewendet werden können und für die meisten in der Gefäßchirurgie vorkommenden Leistendefekte von ausreichender Größe sind.

\section{Einschränkungen}

Ein Nachteil der vorliegenden Studie ist ihr retrospektiver Charakter. Alle möglichen Nachteile dieses Designs, wie beispielsweise fehlende Daten und Erinnerungsverzerrung, können vorhanden sein. Außerdem ist die Studienpopulation relativ klein. Aufgrund des retrospektiven Charakters dieser Studie erfolgte keine Randomisierung und wir fanden signifikant mehr Patienten mit chronischer Niereninsuffizienz in der Sartorius-Gruppe. Diese unterschiedliche Verteilung eines Risikofaktors für Wundheilungsstörungen kann die Ergebnisse beeinflussen.

\section{Zukunftsperspektiven}

Wie im Vorfeld erörtert, werden für beide Muskellappen zahlreiche potenzielle Vor- und Nachteile beschrieben. Die Entscheidung, welcher Muskellappen zur Bedeckung der Leiste entnommen wird, hängt derzeit hauptsächlich von der Präferenz und Erfahrung des Krankenhauses und des operierenden Chirurgen ab. Die derzeit verfügbaren Daten unterstützen keinen eindeutigen klinischen Vorteil für eines der beiden Verfahren, wobei jedoch keine prospektiven oder randomisiert kontrollierten Studien verfügbar sind. Auch in unserer Studie waren beide Muskellappen gleichwertig im Hinblick auf die klinischen Ergebnisse. In weiteren Studien muss daher untersucht werden, ob eine der beiden Muskellappenplasti- ken der anderen hinsichtlich der klinischen Ergebnisse überlegen ist.

\section{Schlussfolgerung}

Die Muskellappenplastik ist ein effektives Verfahren um Leistendefekte durch tiefe Infektionen nach gefäßchirurgischen Eingriffen zu bedecken, mit guten Ergebnissen in einer schwierigen und verletzlichen Gruppe von Patienten. Es wurden keine Unterschiede zwischen beiden Muskellappenplastiken in Bezug auf Amputationen und Verlust der Gefäßprothese gefunden. Wir empfehlen daher, die Muskellappenplastik abhängig von den Vorlieben und Erfahrungen des Operationsteams durchzuführen. Hierbei ist es jedoch unseres Erachtens sinnvoll, beide Techniken zu beherrschen, da die Rectus-femoris-Muskellappenplastik vor allem zum Bedecken großer Gewebedefekte geeignet ist und die Sartorius-Muskellappenplastik für kleinere Defekte verwendet werden kann.

\section{Fazit für die Praxis}

- Die Muskellappenplastik ist ein effektives Verfahren, um tiefe Infektionen in der Leiste zu behandeln.

- Der Rectus-femoris-Muskel und der Sartorius-Muskel sind gleichwertig hinsichtlich Amputationsraten und Gefäßprothesenverlust.

- Beide Techniken können sicher durchgeführt werden, je nach Präferenz und Erfahrung des Operationsteams.

\section{Korrespondenzadresse}

\section{B. M. E. Mees, MD, PhD}

Klinik für Gefäßchirurgie, Universitätsklinik Maastricht

P. Debyelaan 25, 6229 HX Maastricht, Niederlande

barend.mees@mumc.nl

\section{Einhaltung ethischer Richtlinien}

Interessenkonflikt. L.F. Wübbeke, P.R. Keschenau, D. Kotelis, J.W.H.C. Daemen, M.J. Jacobs und B.M.E. Mees geben an, dass kein Interessenkonflikt besteht.

Für diesen Beitrag wurden von den Autoren keine Studien an Menschen oder Tieren durchgeführt.
Für die aufgeführten Studien gelten die jeweils dort angegebenen ethischen Richtlinien.

Open Access Dieser Artikel wird unter der Creative Commons Namensnennung 4.0 International Lizenz (http://creativecommons.org/licenses/by/4.0/deed. de) veröffentlicht, welche die Nutzung, Vervielfältigung, Bearbeitung, Verbreitung und Wiedergabe in jeglichem Medium und Format erlaubt, sofern Sie den/die ursprünglichen Autor(en) und die Quelle ordnungsgemäßnennen, einen Linkzur Creative Commons Lizenz beifügen und angeben, ob Änderungen vorgenommen wurden.

\section{Literatur}

1. Yeager RA, Porter JM (1992) Arterial and prosthetic graft infection. Ann Vasc Surg 6:485-491

2. Verma H, Ktenidis K, George RK, Tripathi R (2015) Vacuum-assisted closure therapy for vascular graft infection (Szilagyi grade III) in the groin-a 10-year multi-center experience. Int Wound J 12:317-321

3. Turtiainen J, Saimanen E, Partio T et al (2010) Surgical wound infections after vascular surgery: prospective multicenter observational study. Scand J Surg 99:167-172

4. Nguyen LL, Brahmanandam S, Bandyk DF et al (2007) Female gender and oral anticoagulants are associated with wound complications in lower extremity vein bypass: an analysis of 1404 operations for critical limb ischemia. J Vasc Surg 46:1191-1197

5. Chang JK, Calligaro KD, Ryan S et al (2003) Risk factors associated with infection of lower extremity revascularization: analysis of 365 procedures performed at a teaching hospital. Ann Vasc Surg 17:91-96

6. Lee ES, Santilli SM, Olson MM et al (2000) Wound infection after infrainguinal bypass operations: multivariate analysis of putative risk factors. Surg Infect 1:257-263

7. Mirzabeigi MN, Fischer JP, Basta MN et al (2017) Managing groin wounds after infrainguinal vascular procedures: examining the reoperative events and complication profile of muscle flap reconstruction. Ann Vasc Surg 43:232-241

8. Williams IM, Milling MA, Shandall AA (2003) Vascularised muscular flaps and arterial graft infection in the groin. Eur J Vasc Endovasc Surg 25:390-395

9. Szilagyi DE, Smith RF, Elliott JP, Vrandecic MP (1972) Infection in arterial reconstruction with synthetic grafts. Ann Surg 176:321-333

10. Kimmel RM, Murphy RX Jr, Chowdary RP (1994) Optimal management of inguinal vascular graft infections. Ann Plast Surg 32:623-629

11. Perler BA, Kolk CA, Manson PM et al (1993) Rotationalmuscleflaps totreatlocalized prosthetic graft infection: long-term follow-up. J Vasc Surg 18:358-364

12. Ger R (1976) The coverage of vascular repairs by muscle transposition. JTrauma 16:974-978

13. Fischer JP, Mirzabeigi MN, Sieber BA et al (2013) Outcome analysis of 244 consecutive flaps for managing complex groin wounds. J Plast Reconstr Aesthet Surg 66:1396-1404

14. Wu LC, Djohan RS, Liu TS et al (2006) Proximal vascular pedicle preservation for sartorius muscle flap transposition. Plast Reconstr Surg 117:253-258

15. Silvestre L, Pedro LM, Fernandes EFR et al (2015) Rectus femoris muscle flap based on proximal insertion mobilization to cover a groin infected vascular graft. J Vasc Surg 62:1064-1067 


\section{Originalien}

16. Seify H, Moyer HR, Jones GE et al (2006) The role of muscle flaps in wound salvage after vascular graft infections: the Emory experience. Plast Reconstr Surg 117:1325-1333

17. Statista (2018) Durchschnittliche Verweildauer in deutschen Krankenhäusern in den Jahren 1992 bis 2017 (in Tagen). https://de.statista.com/statistik/ daten/studie/2604/umfrage/durchschnittlicheverweildauer-im-krankenhaus-seit-1992/.Zugegriffen: 1. Apr. 2019

18. De Staat van Volksgezondheid en Zorg (2018) Ligduur in ziekenhuizen. https://www.staatvenz. $\mathrm{nl} /$ kerncijfers/ligduur-ziekenhuizen. Zugegriffen: 1. Apr. 2019

19. Colwell AS, Donaldson MC, Belkin M et al (2004) Management of early groin vascular bypass graft infections with sartorius and rectus femoris flaps. Ann Plast Surg 52:49-53

20. Alkon JD, Smith A, Losee JE et al (2005) Management of complex groin wounds: preferred use of the rectus femoris muscle flap. Plast Reconstr Surg 115:776-783

21. Sbitany H, Koltz PF, Girotto JA et al (2010) Assessment of donor-site morbidity following rectus femoris harvest for infrainguinal reconstruction. Plast Reconstr Surg 126:933-940

22. Daigeler A, Dodic T, Awiszus F et al (2005) Donorsite morbidity of the pedicled rectus femoris muscle flap. Plast Reconstr Surg 115:786-792

23. Gardetto A, Raschner C, Schoeller T et al (2005) Rectus femoris muscle flap donor-site morbidity. Br JPlast Surg 58:175-182
24. Yang D, Morris SF, Sigurdson L (1998) The sartorius muscle: anatomic considerations for reconstructive surgeons. Surg Radiol Anat 20:307-310

25. Khalil IM, Sudarsky L (1987) Sartorius muscle "twist" rotation flap: an answer to flap necrosis. JVasc Surg 6:93-94

26. Topel I, Betz T, Uhl C, Steinbauer MG (2011) The impact of Superficial Femoral Artery (SFA) occlusion on the outcome of proximal sartorius muscle transposition flaps in vascular surgery patients. J Vasc Surg 53:1014-1019

27. Dua A, Rothenberg KA, Lavingia K et al (2018) Outcomes of gracilis muscle flaps in the management of groin complications after arterial bypass with prosthetic graft. Ann Vasc Surg 51:113-118

28. Combs PD, Sousa JD, Louie O et al (2014) Comparison of vertical and oblique rectus abdominis myocutaneous flaps for pelvic, perineal, and groin reconstruction. Plast Reconstr Surg 134:315-323

29. Zoepke SN, de Weerd L (2017) The marriage of sartorius and tensor fasciae latae in treating vascular prosthetic graft infections. Plast Reconstr Surg Glob Open 5:e1274

30. Cedidi CC, Felmerer G, Berger A (2005) Management of defects in the groin, thigh, and pelvic region with modified contralateral TRAM/VRAM flaps. Eur JMed Res 10(12):515-520 (Dec)

\section{Hier steht eine Anzeige.}

\section{算 Springer}

